\title{
INTERPOLATION AND DENOISING OF NONUNIFORMLY SAMPLED DATA USING WAVELET-DOMAIN PROCESSING
}

\author{
Hyeokho Choi and Richard Baraniuk
}

Department of Electrical and Computer Engineering, Rice University, Houston, TX 77251-1892, USA

\begin{abstract}
In this paper, we link concepts from nonuniform sampling, smoothness function spaces, interpolation, and denoising to derive a suite of multiscale, maximum-smoothness interpolation algorithms. We formulate the interpolation problem as the optimization of finding the signal that matches the given samples with smallest norm in a function smoothness space. For signals in the Besov space $B_{q}^{\alpha}\left(L_{p}\right)$, the optimization corresponds to convex programming in the wavelet domain; for signals in the Sobolev space $W^{\alpha}\left(L_{2}\right)$, the optimization reduces to a simple weighted least-squares problem. An optional wavelet shrinkage regularization step makes the algorithm suitable for even noisy sample data, unlike classical approaches such as bandlimited and spline interpolation.
\end{abstract}

\section{INTRODUCTION}

The problem of signal reconstruction from nonuniformly sampled data arises in many contexts, including sampling systems with sampling jitter, the design of irregularly spaced antenna arrays, the reconstruction of signals from missing samples, and the processing of geophysical data. In higher dimensions, interpolation from non-Cartesian coordinates is central to many important imaging problems, such as tomography, synthetic aperture radar, and radio astronomy. Computer graphics, in addition, must routinely process nonuniformly distributed samples of images and volumes.

The signal interpolation problem is ill-posed in that there exist an infinite number of continuous-time functions passing through a given set of samples. Hence, interpolation must be formulated as an optimization problem with side constraints to narrow down the class of candidate solutions.

One popular reconstruction approach is bandlimited interpolation: we seek the bandlimited signal of minimum energy that passes through the samples [1-4]. In this case, the optimal minimum-mean-square-error solution is known [2], and regularization schemes have been developed to improve the problem's numerical conditioning [3,4]. Roughly speaking, bandlimited interpolation attempts to favor smooth interpolations by hard-limiting their high-frequency content.

Unfortunately, many important signals and images (finitelength signals and signals containing edges, for instance) cannot be properly modeled as bandlimited. Furthermore, such signals are often nonstationary.

This work was supported by the National Science Foundation, grant MIP-9457438, DARPA/AFOSR, grant F49620-97-1-0513, Texas Instruments, and the Rice Consortium for Computational Seismic Interpretation.

Email: choi@ece.rice.edu, richb@rice.edu

Internet: www.dsp.rice.edu
In this paper, we formulate a new approach to signal interpolation from nonuniform samples. In multiscale maximumsmoothness interpolation we select the signal passing through the samples that has minimum smoothness norm (hence maximum smoothness) in an appropriate function space. Unlike the space of bandlimited functions, smoothness spaces such as Sobolev and Besov spaces contain life-like signals with discontinuities and nonstationarities. Since these function spaces are simply parameterized in the wavelet domain [5], our interpolation algorithm reduces to straightforward processing of the wavelet coefficients of the signal samples. As a bonus, we will derive a simple yet powerful multiscale regularization scheme for interpolating noisy signals that reduces to simple thresholding of the wavelet coefficients.

For signals containing singularities, wavelet-based interpolation offers real performance advantages. Figure 1 demonstrates the supremacy of wavelet-based interpolation over bandlimited and spline interpolation for Donoho's HeaviSine signal [6], which contains both smooth and edgy regions.

After reviewing the relevant theory on wavelets and smoothness spaces in Section 2, we present our algorithm in Sections 3 and 4 . Section 5 provides several illustrative examples, while Section 6 introduces regularization. We close in Section 7 with conclusions and perspectives on future work.

\section{WAVELETS AND SMOOTHNESS SPACES}

The discrete wavelet transform (DWT) represents a 1-D, continuous-time signal $f$ in terms of shifted versions of a lowpass scaling function $\phi$ and shifted and dilated versions of a prototype bandpass wavelet function $\psi$ [7]. For special choices of $\phi$ and $\psi$, the functions $\phi_{j, k}(t) \equiv 2^{j / 2} \phi\left(2^{j} t-k\right), \psi_{j, k}(t) \equiv$ $2^{j / 2} \psi\left(2^{j} t-k\right), j, k \in \mathbb{Z}$ form an orthonormal basis for $L_{2}{ }^{1}$ and we have the representation [7]

$$
f(t)=\sum_{k} u_{j 0, k} \phi_{j 0, k}(t)+\sum_{j=j_{0}}^{\infty} \sum_{k} w_{j, k} \psi_{j, k}(t),
$$

with

$$
u_{j, k} \equiv \int f(t) \phi_{j, k}^{*}(t) d t, \quad w_{j, k} \equiv \int f(t) \psi_{j, k}^{*}(t) d t .
$$

The scale $j_{0}$ represents the coarsest scale under consideration; the $u_{j_{0}, k}$ 's correspond to local means at this scale. We can easily construct 2-D wavelets from the 1-D $\phi$ and $\psi$ [7].

\footnotetext{
${ }^{1}$ In this paper, we consider only functions defined on a finite interval $I$. Define the $L_{p}(I)$ space as the set of all functions on $I$ with bounded norm $\|f\|_{p}^{p} \equiv \int_{I}|f(t)|^{p} d t$
} 


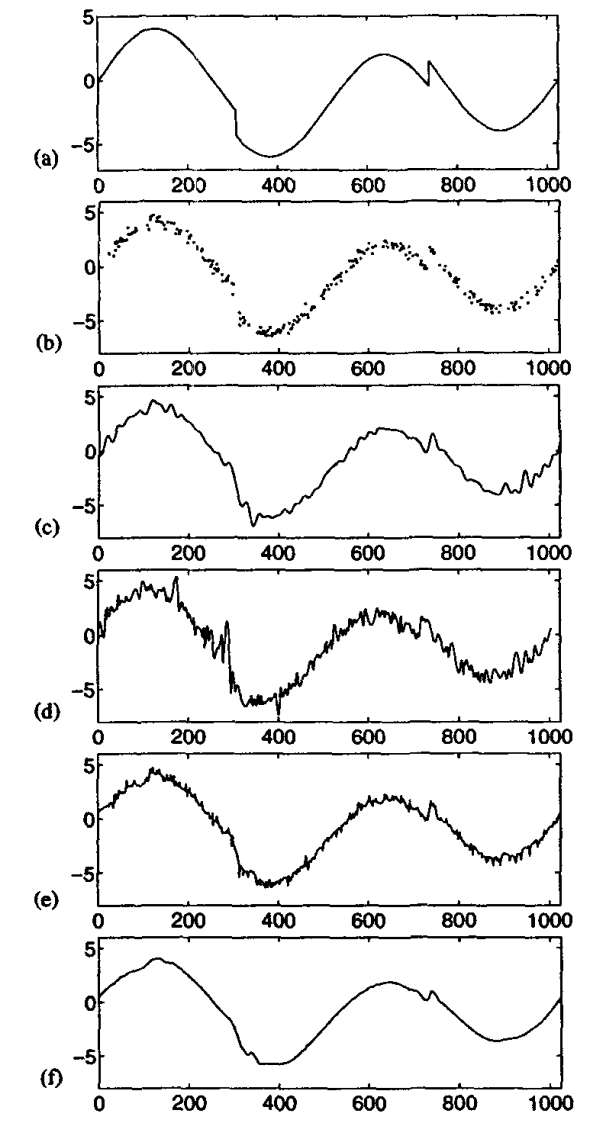

Figure 1: Interpolation of 256 noisy nonuniform samples of the HeaviSine signal. (a) Original signal. (b) 256 nonuniform samples with additive white Gaussian noise of variance $\sigma=0.3$. Results from (c) regularized optimal bandlimited interpolation [3], (d) cubic spline interpolation, (e) maximum-smoothness interpolation, $(f)$ regularized maximum-smoothness interpolation based on wavelet thresholding.

For finite, discrete data, an orthonormal discrete wavelet transformation matrix $\mathbf{W}$ that takes the data vector to a wavelet/scaling coefficient vector can be constructed by "periodization" [8]. Since $\mathbf{W}$ is orthonormal, we have $\mathbf{W}^{-1}=\mathbf{W}^{T}$. Given a finite-length, continuous-time signal $f(t), t \in I=[0,1]{ }^{2}$ define $f$ as the column vector of length $M \equiv 2^{J}$ whose elements are the dyadic samples $f_{i} \equiv f\left(i / 2^{J}\right), i=0, \ldots, M-1$ at the starting (finest) scale $J>j_{0}$. That is, $\mathbf{f} \equiv\left[f_{0}, \ldots, f_{M-1}\right]^{T}$. In general, the elements of the vector Wf will not equal the wavelet coefficients of the continuous time-signal $f(t)$ unless the signal samples are prefiltered $[8,9]$. Denoting the prefiltering matrix by $\mathbf{P}$, the DWT coefficients for scales $j<J$ can be written as $\mathbf{w}=$ WPf. Conversely, the correct continuous-time signal samples can be obtained from the inverse wavelet matrix transformation by "postfiltering." Denote the postfilter matrix as $\mathbf{F}$. Then, we have $\mathbf{f}=\mathbf{F W}^{T} \mathbf{w}$. Define

\footnotetext{
${ }^{2}$ Without loss of generality, we assume that $I=[0,1]$.
}

$\mathbf{V} \equiv \mathbf{F W}^{T}$

Wavelets provide a simple characterization for a wide variety of function smoothness spaces [5]. The norms of these spaces measure signal smoothness: smaller norms imply smoother functions. The scale of Besov spaces $B_{q}^{\alpha}\left(L_{p}(I)\right), 0<\alpha<\infty$, $0<p \leq \infty, 0<q \leq \infty$, are particularly useful, for they contain many life-like signals. For analyzing $\phi$ and $\psi$ possessing $r>\alpha$ vanishing moments [8], the Besov norm $\|f\|_{B_{q}^{\alpha}\left(L_{p}(I)\right)}$ can be defined as a sequence norm on the wavelet coefficients of $f$

$$
\begin{aligned}
\|f\|_{B_{q}^{\alpha}\left(L_{p}(I)\right) \equiv} \equiv & \left\|u_{j_{0}, k}\right\|_{p}^{p}+ \\
& \left(\sum_{j \geq j 0}\left(\sum_{k} 2^{\alpha j p} 2^{j(p-2)}\left|w_{j, k}\right|^{p}\right)^{q / p}\right)^{1 / q} .
\end{aligned}
$$

The three hyperparameters have natural interpretations: a $p$-norm of the wavelet coefficients is taken within each scale $j$, a $q$-norm is taken across scale, and the smoothness parameter $\alpha$ thus controls the rate of decay of the $w_{j, k}$ across scale (frequency). Very roughly speaking, the parameter $\alpha$ corresponds to the number of wellbehaved derivatives of $f$; hence, the larger the $\alpha$, the smoother the functions in $B_{q}^{\alpha}\left(L_{p}(I)\right)[5,11]$. Do not be misled by the terminology "smoothness space" - for $\alpha<1$, Besov spaces contain discontinuous functions.

A simple but useful set of Besov spaces are the Sobolev spaces, obtained as $W^{\alpha}\left(L_{2}(I)\right) \equiv B_{2}^{\alpha}\left(L_{2}(I)\right)$ with $p=q=2$. In the wavelet domain, we have

$$
\|f\|_{W_{2}^{\alpha}\left(L_{2}(I)\right)} \equiv\left\|u_{j_{0}, k}\right\|_{2}^{2}+\left(\sum_{j \geq j_{0}, k}\left|2^{\alpha j} w_{j, k}\right|^{2}\right)^{1 / 2} .
$$

Note that $W^{0}\left(L_{2}(I)\right)=L_{2}(I)$.

\section{MAXIMUM-SMOOTHNESS INTERPOLATION}

The problem of signal interpolation from nonuniformly sampled data can be stated as follows. Let $f(t)$ be the signal under consideration. In practical applications, only a finite segment of the signal is available. Here, we assume that $f(t)$ is defined on the finite interval $I=[0,1]$. In addition, let $\left\{t_{1}, \ldots, t_{N}\right\}$ be the (nonuniform) sampling points on the interval, where $N$ is the number of samples. Without loss of generality, we assume that $0 \leq t_{1}<t_{2}<\cdots<t_{N} \leq 1$. The available data are the sample values at the sampling points, that is, $\left\{f\left(t_{1}\right), \ldots, f\left(t_{N}\right)\right\}$.

The problem is to estimate the original continuous-time signal $f$ from the given nonuniform samples according to some optimality criterion. When the given signal samples are noisy, the signal estimation problem consists of both interpolation and denoising. In our algorithm, the optimality criterion will be the Besov norm of the reconstructed signal, which we will minimize in order to maximize the smoothness of the reconstruction.

Define the dyadic points at scale $j$ to be $\left\{i / 2^{j}, i=\right.$ $\left.0,1, \ldots, 2^{j}-1\right\}$. Define the scale- $j$ dyadic intervals $I_{i} \equiv$ $\left[i / 2^{j},(i+1) / 2^{j}\right)$. To simplify the derivation, we will approximate the set of sampling points $\left\{t_{1}, \ldots, t_{N}\right\}$ with a subset of dyadic points at a certain scale $j=J$. That is, let us assume that $t_{i}=n_{i} / 2^{J}$ for $i=1, \ldots, N$. The error resulting from this approximation depends on the regularity of the signal and the scale $J .^{3}$ The starting scale $J$ should be chosen large enough to make

\footnotetext{
${ }^{3}$ If $|f(t)-f(\nu)| \leq K|t-\nu|^{\beta}$, the approximation error is $O\left(2^{-\beta J}\right)$.
} 
the dyadic approximation of sampling points accurate, as long as the size of the resulting interpolation problem is manageable.

Being given samples of the signal only at the points $t_{i}$ necessarily means that we have no information at other dyadic points. In terms of the wavelet transform, this means that we have no knowledge of the scaling coefficients at points other than $t_{i}$.

Denote the $i$ th row of the matrix $\mathbf{V}$ as $\mathbf{v}_{i}^{T}$. Collecting the rows of the equation $\mathbf{f}=\mathbf{V w}$ only for those indices for which $f_{i}$ is known, we can write the the following constraint equation on the wavelet coefficients $w$

$$
\begin{aligned}
& \quad \mathbf{S w}=\mathbf{f}^{N} \\
& \text { with } \mathbf{S}=\left[\mathbf{v}_{n_{1}}^{T}, \mathbf{v}_{n_{2}}^{T}, \ldots, \mathbf{v}_{n_{N}}^{T}\right]^{T} \text { and } \\
& \mathbf{f}^{N}=\left[f_{n_{1}}, f_{n_{2}}, \ldots, f_{n_{N}}\right]^{T} \text {. } \\
& \text { Because }(1) \text { is an underdetermined system of equations, there } \\
& \text { exist many different solutions for } \mathbf{w} \text { that match the given sampled } \\
& \text { data } \mathbf{f}^{N} \text {. For the minimum Besov solution, we choose the w that } \\
& \text { both satisfies (1) and minimizes }\|f\|_{B_{q}^{\alpha}\left(L_{p}(I)\right) \text {. }} \\
& \text { Because of the simple characterization of Besov norms in } \\
& \text { terms of the wavelet coefficients of } f \text {, we can repose this interpo- } \\
& \text { lation problem as a wavelet-domain optimization problem. For a } \\
& \text { general Besov space } B_{q}^{\alpha}\left(L_{p}(I)\right) \text {, the problem of finding the signal } \\
& \text { that obeys (1) while minimizing }\|f\|_{B_{q}^{\alpha}\left(L_{p}(I)\right)} \text { becomes a nonlin- } \\
& \text { ear constrained optimization problem in } \mathbb{R}^{N}[10] \text {. For } p, q \geq 1 \text {, } \\
& \text { this is a convex functional with linear constraints optimization } \\
& \text { (while simple, for large } N \text { the computational cost of this mini- } \\
& \text { mization could be nontrivial). For Sobolev spaces, however, the } \\
& \text { solution is even more straightforward. }
\end{aligned}
$$

\section{INTERPOLATION IN SOBOLEV SPACE}

In the Sobolev spaces $W^{\alpha}\left(L_{2}(I)\right)$, maximum-smoothness interpolation reduces to simple weighted least-squares optimization in the wavelet domain. Since this case provides many insights into the more general Besov space interpolation problem, we will emphasize it here.

Let $\mathbf{w}=\left[w_{1}, w_{2}, \ldots, w_{M}\right]^{T}$ be the wavelet coefficients of the samples and let $j_{i}, i=1, \ldots, M$, denote the scale of the wavelet coefficients $w_{i}, i=1, \ldots, M$. Denoting the $i$ th column of the matrix $\mathbf{S}$ by $\mathbf{s}_{i}$, we obtain the following equation by weighting the columns of $\mathbf{S}$ and the corresponding elements of $\mathbf{w}$ :

$$
\left[2^{-\alpha j_{1}} \mathbf{s}_{1}, 2^{-\alpha j_{2}} \mathbf{s}_{2}, \ldots, 2^{-\alpha j_{M}} \mathbf{s}_{M}\right]\left[\begin{array}{c}
2^{\alpha j_{1}} w_{1} \\
2^{\alpha j_{2}} w_{2} \\
\vdots \\
2^{\alpha j_{M}} w_{M}
\end{array}\right]=\mathbf{f}^{N}
$$

Let $\widetilde{\mathbf{S}}=\left[2^{-\alpha j_{1}} \mathbf{s}_{1}, 2^{-\alpha j_{2}} \mathbf{s}_{2}, \ldots, 2^{-\alpha j_{M}} \mathbf{s}_{M}\right]$. Then, the leastsquares solution of (2) can be written as $\widetilde{\mathbf{S}}^{T}\left(\widetilde{\mathbf{S}} \widetilde{\mathbf{S}}^{T}\right)^{-1} \mathbf{f}^{N}$, and the the solution to the original problem is obtained by restoring the weighting:

$$
\widetilde{\mathbf{w}}=\operatorname{diag}\left\{2^{-\alpha j_{1}}, \ldots, 2^{-\alpha j_{M}}\right\} \widetilde{\mathbf{S}}^{T}\left(\widetilde{\mathbf{S}} \widetilde{\mathbf{S}}^{T}\right)^{-1} \mathbf{f}^{N} .
$$

The desired time-domain interpolation can be obtained by inverse wavelet transform of $\widetilde{\mathbf{w}}$. Ignoring the error in dyadic approximation, the interpolated signal is maximally smooth in the sense of Sobolev norm among all functions passing through the samples. (a)

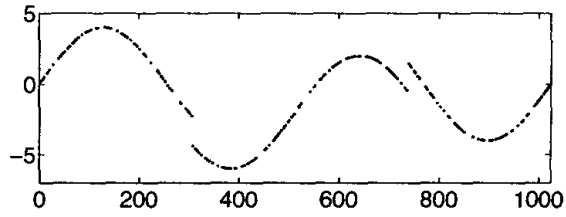

(b)

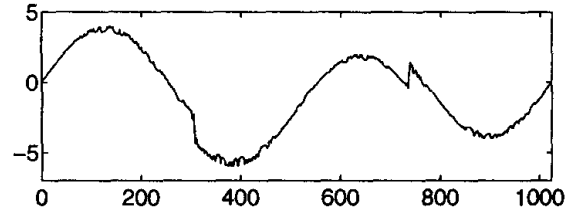

(c)

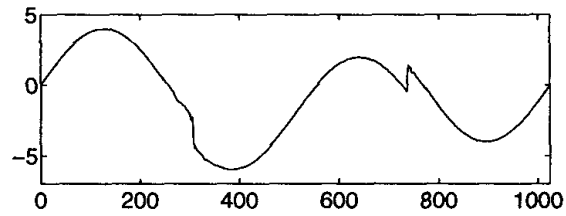

Figure 2: Maximum-smoothness interpolation of 256 nonuniform samples of the HeaviSine signal. (a) 256 nonuniform samples. Maximum-smoothness interpolation with (b) $\alpha=0.5$ and (c) $\alpha=$ 0.9 .

\section{EXAMPLES}

To illustrate the behavior of the maximum smoothness interpolator for both smooth and irregular signal regions, we choose Donoho's HeaviSine signal [6] for the test.

For a discrete realization of the interpolation algorithm, we approximate the underlying continuous-time signal by its uniform samples at 1024 points. For signals living in the interval $I=[0,1]$, this corresponds to assuming that the finest scale is $J=10$. In order to eliminate the error due to dyadic approximation to isolate the behavior of the interpolation algorithm, we assume that the set of nonuniform sampling points is a subset of the $2^{J}$ dyadic points. In all cases we employed the $D 8$ wavelet [7].

Figure 2 illustrates the performance of the interpolator when the nonuniform samples are relatively dense. We randomly chose $N=256$ of the 1024 dyadic points as the nonuniform sampling points. In the Figure we plot the 256 nonuniform samples, and reconstructions using maximum smoothness interpolation with $\alpha=$ 0.5 and 0.9 . For a properly chosen $\alpha$, the maximum-smoothness interpolation approximates the original signal very well. Note how larger $\alpha$ results in more signal smoothing.

Figure 3 illustrates the performance of the interpolator when the sampling density is low. We randomly chose $N=32$ of the 1024 dyadic points as the nonuniform sampling points. In the Figure we plot the 32 nonuniform samples, and reconstructions by maximum smoothness interpolation with $\alpha=0.8$ and 2.0. Again, we see that the smoothness of the interpolated signal increases with $\alpha$. Because the sampling density here is low, we must impose more regularity in order to connect the given sample points. Sampling destroys the details of the original signal, so it is natural for the interpolated signal to be smoother than the original. However, with our multiscale algorithm, the interpolated signal retains the discontinuities of the original signal. This is a clear advantage over usual bandlimited signal interpolation, where the discontinuities are necessarily smoothed. 
(a)

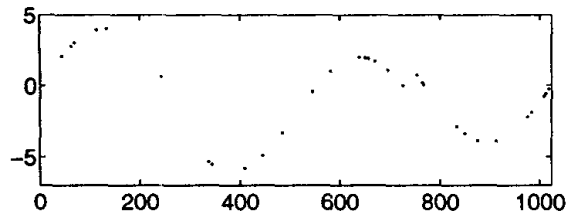

(b)

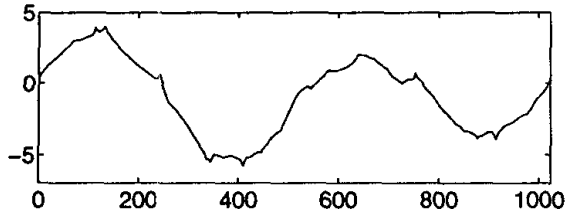

(c)

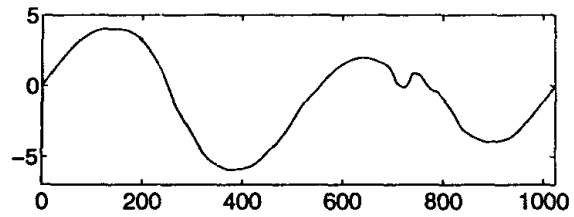

Figure 3: Maximum-smoothness interpolation of 32 nonuniform samples of the HeaviSine signal. (a) 32 nonuniform samples. Maximum-smoothness interpolation with (b) $\alpha=0.8$ and (c) $\alpha=$ 2.0.

\section{REGULARIZATION VIA WAVELET DENOISING}

When noise corrupts the signal samples, maximum-smoothness interpolation may not satisfactory. See, for example, Figure 1 where we interpolate under the same conditions as in Figure 2 except with samples corrupted by additive white Gaussian noise.

Although the interpolated signal becomes smoother as we increase $\alpha$, we can never completely remove the noisy character of the interpolated signal. This is because the interpolated signal must necessarily pass through all sample points, even when the sample values are noisy. Hence, the smoothest function matching noisy samples can be very irregular (with large smoothness norm).

Regularization can be employed to yield smoother interpolations at the expense of errors in matching the given sample points. The regularization of functions in Besov space can be formulated as a variational problem in the wavelet domain [11]. Astonishingly, in many cases regularization can be accomplished simply by shrinking the signal wavelet coefficients. Figure 1(f) shows the signal obtained by hard thresholding the wavelet coefficients of the signal in (e) with threshold $\tau=0.7$ before inverting the wavelet transform. By proper choice of the threshold, the sampling noise can be suppressed at the expense of only minimal distortion in the estimated signal.

Other interpolation algorithms do not have this joint interpolation/regularization capability. Bandlimited interpolation leaves much to be desired, because the bandlimit cannot be set appropriately: A large bandlimit results in noisy interpolation (see Figure 1(c)), while a small bandlimit oversmooths the signal. Even worse, cubic spline interpolations are extremely noisy, especially when the sampling is very irregular (see Figure 1(d)).

The choice of the regularization parameter (wavelet threshold) depends on the properties desired in the underlying signal. Unfortunately, the interpolated signal obtained by minimum-smoothness norm interpolation is difficult to characterize, even if the noise in the signal samples is white Gaussian.

\section{CONCLUSIONS}

In this paper, we have demonstrated the efficacy of a new multiscale maximum-smoothness interpolator for both signal reconstruction and joint reconstruction/denoising. Our algorithm is straightforward, owing to the remarkably simple characterization of the Sobolev and Besov smoothness spaces by wavelets. In fact, for Sobolev space, the entire algorithm reduces to a simple leastsquares problem in the wavelet domain. Extension to higher dimensions (for image and volume data) is trivial.

Investigation of the behavior of maximum smoothness interpolator for other smoothness spaces remains a topic of future research. Currently, we are developing methods to automatically set the smoothness and regularization parameters for a given problem and investigating the accuracy of the dyadic sampling approximation in terms of signal regularity.

\section{REFERENCES}

[1] A. J. Jerry, "The Shannon sampling theorem - its various extensions and applications: A tutorial review," Proc. IEEE, vol 65, pp. 1565-1596, November 1977.

[2] J. L. Yen, "On nonuniform sampling of bandlimited signals," IRE Trans. Circuit Theory, vol. CT-3, pp. 251-257, Dec. 1956.

[3] E. J. Diethorn and D. C. Munson, Jr., "A linear, time-varying system framework for noniterative discrete-time band-limited signal extrapolation," IEEE Trans. Signal Proc., vol. 39, pp. 55-68, Jan. 1991.

[4] H. Choi and D. C. Munson, Jr., "Analysis and design of minimax-optimal interpolators," IEEE Trans. Signal Proc., vol. 46, no. 6, pp. 1571-1579, June 1998.

[5] Y. Meyer, Wavelets and Operators. Cambridge Univ. Press, 1992.

[6] D. Donoho and I. Johnstone, "Adapting to unknown smoothness via wavelet shrinkage," J. Amer. Statist. Assoc., vol. 90 , pp. 1200-1224, 1995.

[7] I. Daubechies, Ten Lectures on Wavelets. New York: SIAM, 1992.

[8] G. Strang and T. Nguyen, Wavelets and Filter Banks. Wellesley, MA: Wellesley-Cambridge Press, 1996.

[9] P. Abry and P. Flandrin, "On the initialization of the discrete wavelet transform algorithm," IEEE Signal Proc. Letters, vol. 1, no. 2, pp. 32-34, Feb. 1994.

[10] D. G. Luenberger, Linear and Nonlinear Programming. Reading, MA: Addison-Wesley Publishing, 1984.

[11] A. Chambolle, R. A. DeVore, N. Lee, and B. J. Lucier, "Nonlinear wavelet image processing: variational problems, compression, and noise removal through wavelet shrinkage," IEEE Trans. on Image Proc., vol. 7, no. 3, pp. 319-335, March, 1998. 